\title{
Effects of chronic kidney disease on mid- and long- term clinical outcomes after off-pump coronary artery bypass grafting-a retrospective study
}

Xihui Li ( $\square$ heart2000@163.com )

Peking University First Hospital https://orcid.org/0000-0003-0268-2386

\section{Bo Song}

Peking University First Hospital

\section{Shiyong Dong}

Peking University First Hospital

Siyu Zhang

Peking University First Hospital

Research article

Keywords: chronic kidney disease, coronary artery bypass grafting, off-pump, mid-long-term follow-up, prognosis

Posted Date: July 6th, 2020

DOl: https://doi.org/10.21203/rs.3.rs-39683/v1

License: (9) (1) This work is licensed under a Creative Commons Attribution 4.0 International License. Read Full License 


\section{Abstract}

Backgroud: To investigate the effect of chronic kidney disease (CKD) on mid- and long-term clinical outcomes after off-pump coronary artery bypass grafting (CABG).

Methods: This was a retrospective analysis of data of 1141 patients discharged from the Department of Cardiac Surgery, Peking University First Hospital from January 2010 to June 2018 after undergoing offpump CABG. Preoperative baseline, operative, and follow-up data obtained at regular outpatient visits or by telephone calls were collected. Follow-up endpoints included stroke, nonfatal myocardial infarction, heart failure, revascularization, and all-cause death. Patients with preoperative estimated glomerular filtration rate calculated, using the Chronic Kidney Disease Epidemiology equation of $\geq 60 \mathrm{~mL} / \mathrm{min} / 1.73$ $\mathrm{m}^{2}$ and $<60 \mathrm{~mL} / \mathrm{min} / 1.73 \mathrm{~m}^{2}$, were assigned to normal Group (1, 910 cases) and CKD group (231 cases), respectively. The effects of CKD on selected endpoint events were compared and analyzed.

Results: There was a higher proportion of women, more preoperative complications, and a higher incidence of early postoperative complications in patients with CKD than in those with normal renal function. After 1-9 years of follow-up (mean5.0 \pm 2.2 years), the incidences of stroke, non-fatal myocardial infarction, and all-cause mortality were significantly higher in the CKD than in the normal renal function group, whereas incidences of revascularization and heart failure were not. Logistic regression analysis showed that preoperative CKD was a risk factor for stroke, non-fatal myocardial infarction, and all-cause death during follow-up. After correcting for common confounding factors, such as sex, age, and left ventricular ejection fraction, preoperative CKD was a risk factor for non-fatal myocardial infarction (OR 2.675, 95\% $\mathrm{Cl} 1.023-6.995, \mathrm{P}=0.045)$ and all-cause death $(\mathrm{OR} 1.833,95 \% \mathrm{Cl}$ $1.079-3.114, P=0.025)$.

Conclusions: In patients undergoing off-pump CABG, preoperative CKD is associated with increases in the incidences of mid- and long-term non-fatal myocardial infarction and all-cause mortality.

\section{Background}

Chronic kidney disease (CKD) is a major risk factor for coronary artery disease (CAD) (1), and the risk of death, cardiovascular events, and hospitalization increase with decreasing estimated glomerular filtration rate (EGFR)(2).Coronary artery bypass grafting(CABG) and percutaneous coronary intervention (PCI) are effective treatments for severe coronary heart disease. A meta-analysis found that CABG is associated with significantly lower long-term mortality in patients with CKD and end-stage renal disease than is $\mathrm{PCI}$ (3).

Whether off-pump or on-pump CABG is preferable for patients with coronary heart disease with CKD is controversial. Current studies have shown that off-pump CABG may be superior, possibly because offpump obviates the need for cardiopulmonary bypass and protects renal function in the perioperative period. It is also associated with fewer complications, including allogeneic blood transfusion, postoperative thoracotomy hemostasis, acute kidney injury, and respiratory system dysfunction (4).In 
particular, off-pump CABG significantly reduces operative mortality and risk of postoperative dialysis in patients with moderate or severe renal insufficiency (5). Off-pump CABG is currently preferred in mainland China, accounting for approximately $95 \%$ of total CABG procedures in our department. Management of such patients is very complicated, and the impact of the comorbidity of CKD on mid- and long-term clinical outcomes of off-pump CABG has rarely been studied. We therefore conducted a retrospective study of the mid-and long-term outcomes of 1141 patients, who had undergone off-pump CABG from January 2010 to June 2018 in the Department of Cardiac Surgery, Peking University First Hospital.

\section{Methods}

Inclusion criteria were as follows: consecutive, first-time patients undergoing off-pump CABG discharged from our hospital from January 2010 to June 2018. Exclusion criteria were as follows: acute renal insufficiency, preoperative end-stage renal disease dependent on dialysis, intraoperative transfer to onpump, death within 30 days after surgery, incomplete data, and refusal to attend for follow-up. The Chronic Kidney Disease Epidemiology Collaboration (CKD-EPI) equation was used to calculate eGFR (6).Patients with eGFR $\mathrm{CKD}_{\text {EPI }}>60 \mathrm{~mL} / \mathrm{min} / 1.73 \mathrm{~m}^{2}$ were classified as having normal renal function and those with $<60 \mathrm{~mL} / \mathrm{min} / 1.73 \mathrm{~m}^{2}$ as having CKD. Baseline and operative data during hospitalization and postoperative outpatient or telephone follow-up data were collected for statistical analysis. Clinical outcomes included stroke, nonfatal myocardial infarction, heart failure, revascularization, and all-cause death.

SPSS21.0 software(IBM Corp., Armonk, NY, USA) was used for analysis; the $\chi^{2}$ or Fisher's exact test for analyzing count data between two groups and a group $t$-test for measurement data. The effect of preoperative CKD on all clinical outcome events during follow-up was analyzed by logistic regression. Odds ratios (ORs) and $95 \%$ confidence intervals were calculated by univariate analysis and corrected for common confounding factors, such as sex, age, and left ventricular ejection fraction (LVEF). $P<0.05$ was considered to denote a significant difference (two-sided test).

\section{Results}

A total of 1141 patients were included in the study: 910 in the normal Group and 231 in the CKD group. Comparisons of baseline and operative data during hospitalization are shown in Table 1.There was a higher proportion of women, older mean age, and higher incidence of preoperative and postoperative complications in the CKD group than in the normal renal function group.

All patients underwent outpatient or telephone follow-up for 1 -9 years (mean $5.0 \pm 2.2$ years). The incidences of stroke, non-fatal myocardial infarction, and all-cause mortality were significantly higher in the CKD than the normal renal function group, whereas the incidences of revascularization and heart failure were not. Clinical endpoints according to study group are shown in Table 2. 
Results of logistic regression analysis on the effect of preoperative CKD on clinical outcome events are shown in Table 3.Preoperative CKD was found to be a risk factor for stroke, non-fatal myocardial infarction, and all-cause mortality. After correction for common confounding factors, such as sex, age, and LVEF, preoperative CKD was identified as a risk factor for non-fatal myocardial infarction and allcause mortality.

\section{Discussion}

Patients with CKD have a significantly increased risk of coronary heart disease, not only because of traditionally recognized CAD risk factors such as diabetes and hypertension, but also because of other non-traditionally recognized cardiovascular disease risk factors associated with uremia, including inflammation, oxidative stress, and abnormal calcium-phosphorus metabolism (1).Lipid metabolism disorders, mainly hypertriglyceridemia, are risk factors for coronary heart disease in patients with CKD. This accounts for a prevalence of coronary heart disease of approximately $12 \%$ in patients with Stage 3 or greater CKD, compared with only $5 \%$ in those with normal renal function (7).CKD is often accompanied by disorders of mineral bone metabolism, hypocalcemia, hyperphosphatemia, and secondary hyperparathyroidism, all of which can accelerate systemic atherosclerosis and vascular calcification (8).Inflammatory responses, oxidative stress, impaired endothelial cell function, coronary artery calcification, hyperhomocysteinemia, immunosuppression, and other mechanisms can contribute to significant acceleration of atherosclerosis, leading to a poor prognosis in this patient group. Complications of coronary heart disease, such as myocardial infarction and heart failure, are the main causes of death (9).Particularly in dialysis-dependent patients with advanced CKD, the death rate is as high as $40-50 \%$ (10).A 2012 epidemiological survey reported an incidence of CKD in mainland China of $10.8 \%$ and estimated that there were 119.5 million patients with CKD in that country(11).Increasing numbers of these patients will require CABG in the future. There is an urgent need to improve postoperative long-term survival rate.

In patients with severe coronary heart disease and non-dialysis-requiring CKD, CABG achieves significantly lowe rmortality, re-infarction, and revascularization rates than do $\mathrm{PCl}$ and drug therapy (12, 13). In one study 2108 patients with CKD who underwent PCl using drug-eluting stents, $(n=1165)$ or CABG $(n=943)$ were prospectively analyzed in a nonrandomized study with an average follow-up of 41.4 months. Although no significant differences in all-cause death, stroke, and myocardial infarction were observed between the two groups, the revascularization rate was significantly higher in the PCI group (14).A meta-analysis of 29,246 patients enrolled in 11 studies showed that CABG achieved lower longterm all-cause mortality, cardiac mortality, and incidences of myocardial infarction and revascularization, and fewer major adverse cardiac and cerebrovascular events (MACCE) than did drug-eluting stents for revascularization in patients with multivessel coronary heart disease and CKD. However, mortality and risk of cerebrovascular events have recently increased (15).Although CABG can significantly improve the survival rate, it also increases the incidence of postoperative renal failure, especially in high-risk patients (16). 
Off-pump CABG reportedly has a protective effect on renal function in the perioperative period because of it does not entail cardiopulmonary bypass; it also reduces the incidences of complications, such as allogeneic blood transfusion, post-operative thoracotomy hemostasis, acute kidney injury, and respiratory system dysfunction (4).However, in the CORONARY study, no significant difference was observed between on-pump and off-pump CABG in effects on renal function the 1 year follow-up, and off-pump had no demonstrable long-term renal protective effect (17). In the present study, there was a higher proportion of women, older mean age, and higher incidences of preoperative and postoperative complications in the CKD than in the normal group. The follow-up period ranged from 1 to 9 years, with an average of $5.0 \pm 2.2$ years. The incidences of stroke, non-fatal myocardial infarction, and all-cause mortality were significantly higher in the CKD than in the normal renal function group; however, those of revascularization and heart failure were not. Logistic regression analysis showed that preoperative CKD was a risk factor for stroke, non-fatal myocardial infarction, and all-cause mortality. After correcting for common confounding factors such as sex, age, and LVEF, preoperative CKD was identified as a risk factor for non-fatal myocardial infarction and all-cause mortality in the off-pump CABG over a mid- to long-term follow-up.

CKD and end-stage renal disease not only increase the risk of CAD, but also change its clinical manifestations and main symptoms. Management of patients with CAD and CKD is complex because of the possibility of complications and adverse effects during interventions (1).Protection of renal function and improvement in postoperative survival are critical for discharged patients with CKD not undergoing dialysis who have undergone CABG. There is a need for cooperation among cardiac surgeons, cardiologists, nephrologists, and endocrinologists to enhance postoperative management and construct comprehensive strategies, to improve prognosis and reduce complications and mortality in high-risk patients.

First, the identification of high-risk patients facilitates rational allocation of medical resources, targeted prevention, and management. As renal function worsens, the length of hospital stay and medical costs and the number of complications increase. Decreases in creatinine clearance to 80,60 , and $20 \mathrm{~mL} / \mathrm{min}$ reportedly lead to cost increases of $10 \%, 20 \%$, and $30 \%$, respectively. Additionally, the incidence of need for dialysis and mortality increase postoperatively (18).Grouping of CKD severity on the basis of eGFR calculated by the CKD-EPI equation effectively predicts postoperative complications and mortality in both the short-and long-term (23). eGFR ${ }_{\text {CKD-EPI }}<30 \mathrm{~mL} / \mathrm{min} / 1.73 \mathrm{~m}^{2},<41 \mathrm{~mL} / \mathrm{min} / 1.73 \mathrm{~m}^{2},<$ $27 \mathrm{~mL} / \mathrm{min} / 1.73 \mathrm{~m}^{2}$, and $<29 \mathrm{~mL} / \mathrm{min} / 1.73 \mathrm{~m}^{2}$ are predictors of early mortality, stroke, prolonged length of hospital stay, and postoperative dialysis substitution, respectively. Additionally, eGFR $<26 \mathrm{~mL} / \mathrm{min} / 1.73 \mathrm{~m}^{2},<25 \mathrm{~mL} / \mathrm{min} / 1.73 \mathrm{~m}^{2},<35 \mathrm{~mL} / \mathrm{min} / 1.73 \mathrm{~m}^{2}$, and $<29 \mathrm{~mL} / \mathrm{min} / 1.73 \mathrm{~m}^{2}$ are predictors of all-cause death, cardiovascular death, myocardial infarction, and heart failure, respectively, during follow-up (average follow-up time 66 months) (19).Therefore, the management of high-risk patients needs to be further strengthened. 
Second, active treatment of comorbidities such as hypertension, diabetes, and anemia is vital. Hypertension and diabetes are the two commonest comorbidities in patients with CKD.

Recommendations for treatment of diabetic nephropathy include control of $\mathrm{HB} 1 \mathrm{Ac}<7 \%$, blood pressure $<$ 140/90 mmHg, and treatment with renin-angiotensin system blockers (20).Patients with CKD have a high incidence of hypertension that may lead to or originate from CKD. Hypertension aggravates CKD, resulting in uncontrollable hypertension (21).The 2017 American College of Cardiology/American Heart Association Hypertension Guidelines set a BP target of $<130 / 80 \mathrm{~mm} \mathrm{Hg}$ for patients with CKD and other strong cardiovascular risk factors to reduce mortality in patients with CKD. However, few data are available for patients with diabetes or Stage 4-5 CKD (22).Anemia, another common manifestation of renal insufficiency, causes decreased erythropoietin secretion and renal anemia. Anemia predicts the risk of cardiovascular events and death in patients with coronary heart disease (23). It is also an independent risk factor for increased mortality of coronary heart disease in CKD patients with blood hemoglobin below $12 \mathrm{~g} / \mathrm{dL}(24)$.

Third, measures for secondary prevention of coronary heart disease should be actively continued postoperatively, including oral antiplatelet drugs, statin treatment, and good living habits.

In conclusion, patients with CKD undergoing CABG are older preoperatively, have more complications, and more early postoperative complications than those with normal renal function. Mid- and long-term followup after CABG revealed associations between preoperative CKD and higher incidences of nonfatal $\mathrm{MI}$ and all-cause mortality (after correcting for common confounding factors such as sex, age, and LVEF).Among patients with CKD undergoing off-pump CABG, it is necessary to identify high-risk patients and strengthen comprehensive management to reduce complications and improve long-term survival.

\section{Abbreviations}

chronic kidney disease

CKD

coronary artery disease

CAD

coronary artery bypass grafting

CABG

estimated glomerular filtration rate

EGFR

percutaneous coronary intervention

$\mathrm{PCl}$

Chronic Kidney Disease Epidemiology Collaboration

CKD-EPI

left ventricular ejection fraction

LVEF

major adverse cardiac and cerebrovascular events 
MACCE

\section{Declarations}

\section{Ethics Approval and consent to participate}

This study was approved by the biomedical research ethics committee of Peking University First Hospital (approval No. 2018, scientific research 242). And consent was waived.

\section{Consent for Publication}

Not applicable

\section{Availability of supporting data}

The datasets used and/or analysed during the current study are available from the corresponding author on reasonable request.

\section{Competing interests}

The authors have no conflict of interest to declare.

\section{Funding Sources}

We received no funding for this study.

\section{Author Contributions}

Bo Song and Xihui Li Literature search; shiyong Dong and Siyu zhang Data collection. . All authors read and approved the final manuscript.

\section{Acknowledgment}

We thank Dr Trish Reynolds, MBBS, FRACP, from Liwen Bianji, Edanz Group China (www.liwenbianji.cn/ac), for editing the English text of a draft of this manuscript.

\section{References}

1. Sarnak MJ, Amann K, Bangalore S, et al. Chronic kidney disease and coronary artery disease: JACC state-of-the-art review. J Am Coll Cardiol. 2019;74:1823-38.

2. Go AS, Chertow GM, Fan D, et al. Chronic kidney disease and the risks of death, cardiovascular events, and hospitalization. N Engl J Med. 2004;351:1296-305.

3. Kannan A, Poongkunran C, Medina R, et al. Coronary Revascularization in Chronic and End-Stage Renal Disease: A Systematic Review and Meta-analysis. Am J Ther. 2016;23:e16-28. 
4. Lamy A, Devereaux PJ, Prabhakaran D, et al. Off-pump or on-pump coronary-artery bypass grafting at 30 days. N Engl J Med. 2012;366:1489-97.

5. Ueki $\mathrm{C}$, Miyata $\mathrm{H}$, Motomura $\mathrm{N}$, et al. Off-pump technique reduces surgical mortality after elective coronary artery bypass grafting in patients with preoperative renal failure[J]. The Journal of Thoracic Cardiovascular Surgery. 2018;156:976-83.

6. Michels WM, Grootendorst DC, Verduijn M, et al. Performance of the Cockcroft-Gault, MDRD, and new CKD-EPI formulas in relation to GFR, age, and body size. Clin J Am Soc Nephrol. 2010;5:1003-9.

7. Lamprea-Montealegre JA, Mcclelland RL, Grams M, et al. Coronary heart disease risk associated with the dyslipidaemia of chronic kidney disease[J]. Heart. 2018;104:1455-60.

8. Reiss AB, Miyawaki N, Moon J,et al. CKD, arterial calcification, atherosclerosis and bone health: Interrelationships and controversies. Atherosclerosis. 2018;278:49-59.

9. Cai Q, Mukku VK, Ahmad M. Coronary Artery Disease in Patients with Chronic Kidney Disease: A Clinical Update. Current Cardiology Reviews. 2013;9:331-9.

10. US Renal Data System 2012 Annual Data Report Collins AJ, Foley RN, Herzog C, et al. US Renal Data System 2012 Annual Data Report. Am J Kidney Dis. 2013; 61:A7, e1-476.

11. Zhang $L$, Wang $F$, Wang L,et al. Prevalence of chronic kidney disease in China: a cross-sectional survey.Lancet,2012; 379:815-822.

12. Roberts JK, Rao SV, Shaw LK, et al. Comparative Efficacy of Coronary Revascularization Procedures for Multivessel Coronary Artery Disease in Patients with Chronic Kidney Disease. Am J Cardiol. 2017;119:1344-51.

13. Charytan DM, Desai M, Mathur $M$, et al. Reduced risk of myocardial infarct and revascularization following coronary artery bypass grafting compared with percutaneous coronary intervention in patients with chronic kidney disease. Kidney Int. 2016;90:411-21.

14. Kang SH, Lee CW, Yun S-C, et al. Coronary Artery Bypass Grafting vs. Drug-Eluting Stent Implantation for Multivessel Disease in Patients with Chronic Kidney Disease. Korean Circ J. 2017;47:354-60.

15. Wang Y, Zhu S, Gao P, et al. Comparison of coronary artery bypass grafting and drug-eluting stents in patients with chronic kidney disease and multivessel disease: A meta-analysis[J]. European Journal of Internal Medicine, Eur J Intern Med. 2017;43:28-35.

16. Charytan DM, Natwick T, Solid CA, et al. Comparative Effectiveness of Medical Therapy, Percutaneous Revascularization, and Surgical Coronary Revascularization in Cardiovascular Risk Subgroups of Patients With CKD: A Retrospective Cohort Study of Medicare Beneficiaries. Am J Kidney Dis. 2019;74:463-73.

17. Garg AX, Devereaux PJ, Yusuf S, et al. Kidney function after off-pump or on-pump coronary artery bypass graft surgery: a randomized clinical trial. JAMA. 2014;311:2191-8.

18. LaPar DJ, Rich JB, Isbell JM, et al. Preoperative Renal Function Predicts Hospital Costs and Length of Stay in Coronary Artery Bypass Grafting. Ann Thorac Surg. 2016;101:606-12. 
19. Gelsomino S, Del Pace SD, Parise O, et al. Impact of renal function impairment assessed by CKDEPI estimated glomerular filtration rate on early and late outcomes after coronary artery bypass grafting. Int J Cardiol. 2017;227:778-87.

20. Umanath K, Lewis JB. Update on Diabetic Nephropathy: Core Curriculum 2018. Am J Kidney Dis. 2018;71:884-95.

21. Horowitz B, Miskulin D, Zager P. Epidemiology of hypertension in CKD. Adv Chronic Kidney Dis. 2015;22:88-95.

22. ChangAR, Lóser M, MalhotraR, et al. Blood Pressure Goals in Patients With CKD: A Review of Evidence and Guidelines. Clin J Am Soc Nephrol. 2019;14:161-9.

23. Cook JR, Dillie KS, Hakeem A, et al. Effectiveness of Anemia and Chronic Kidney Disease as Predictors for Presence and Severity of Coronary Artery Disease in Patients Undergoing Stress Myocardial Perfusion Study[J]. Am J Cardiol. 2008;102:266-71.

24. Mccullough PA, Lepor NE. Piecing together the evidence on anemia: the link between chronic kidney disease and cardiovascular disease.[J]. Reviews in Cardiovascular Medicine. 2005;6(Suppl 3):4-12.

\section{Tables}


Table 1. Comparison of baseline and operative data between the CKD and nomal

\begin{tabular}{|c|c|c|c|c|}
\hline & $\begin{array}{l}\text { normal Group } 1 \\
\quad(n=910)\end{array}$ & $\begin{array}{l}\text { CKD Group } 2 \\
(n=231)\end{array}$ & $t / X^{2}$ & $p$ \\
\hline Age(ys) & $63.4 \pm 9.2$ & $69.7 \pm 8.9$ & 9.328 & 0.000 \\
\hline Female sex $(\%)$ & $228(25.1)$ & $111(48.1)$ & 46.656 & 0.000 \\
\hline Hypertension(\%) & $595(65.4)$ & $194(84.0)$ & 29.871 & 0.00 \\
\hline Diabetes $(\%)$ & $347(38.1)$ & $120(51.9)$ & 14.546 & 0.000 \\
\hline \multicolumn{2}{|c|}{ Stroke before operation(\%)90(21.1) } & $67(28.5)$ & 6.970 & 0.008 \\
\hline Hyperlipemia(\%) & $384(42.1)$ & $113(47.4)$ & 3.384 & 0.066 \\
\hline $\mathrm{OMI}(\%)$ & $345(37.9)$ & $92(39.8)$ & 0.286 & 0.593 \\
\hline $\operatorname{LVEF}(\%)$ & $61.2 \pm 13.3$ & $57.8 \pm 15.0$ & 3.380 & 0.001 \\
\hline Hemoglobin $(g / L)$ & $135.1 \pm 15.4$ & $123.1 \pm 17.9$ & 10.211 & 0.000 \\
\hline AF before operation( $\%)$ & $40(4.3)$ & $21(8.4)$ & 6.704 & 0.01 \\
\hline $\mathrm{BNP}(\mathrm{pg} / \mathrm{mL})$ & $243 \pm 415$ & $428 \pm 625$ & 4.270 & 0.000 \\
\hline Left main involved(\%) & $246(27.0)$ & $78(33.8)$ & 4.108 & 0.043 \\
\hline Emergency $(\%)$ & $17(1.9)$ & $4(1.7)$ & - & 1.000 \\
\hline LIMA use(\%) & $788(86.6)$ & $169(73.2)$ & 25.693 & 0.000 \\
\hline $\mathrm{LCOS}$ & $58(6.4)$ & $22(9.5)$ & 2.804 & 0.094 \\
\hline IABP use & $58(6.4)$ & $30(13.0)$ & 11.321 & 0.001 \\
\hline Grafts & $2.7 \pm 0.8$ & $2.5 \pm 0.8$ & 2.143 & 0.032 \\
\hline Re-open & $8(0.9)$ & $4(1.7)$ & - & 0.276 \\
\hline Re intubation & $10(1.1)$ & $7(3.0)$ & 4.682 & 0.030 \\
\hline $\mathrm{AKI}$ & $239(26.3)$ & $102(44.2)$ & 28.146 & 0.000 \\
\hline ARDS & $90(9.9)$ & $29(12.6)$ & 1.400 & 0.237 \\
\hline Stroke peri-operative & $13(14.3)$ & $9(3.9)$ & 5.932 & 0.015 \\
\hline UGH & $12(13.2)$ & $9(3.9)$ & 6.774 & 0.029 \\
\hline AFperi-operative & $153(16.8)$ & $52(22.5)$ & 4.058 & 0.044 \\
\hline
\end{tabular}

AF: atria lfibrillation; AKI: acute renal injury; ARDS: acute respiratory distress syndrome; BNP: brain natriuretic peptide; COPD: chronic obstructive pumonary disease; IABP. intra-aortic balloon purnp; LCOS: low cardiac output syndrome; LIMA: left intemal mammary artery; LVEF: left ventricular ejection fraction; OMI: old myocardial infarction; UGH: upper gastrointestinal hemorrhage. 
Table 2. Comparison of clinical outcome events between CKD and normal groups

\begin{tabular}{lcccc}
\hline & $\begin{array}{c}\text { Normal Group } \\
(\mathrm{n}=910)\end{array}$ & $\begin{array}{c}\text { CKD Group 2 } \\
(\mathrm{n}=231)\end{array}$ & $t / X^{2}$ & $p$ \\
\hline Stroke(\%) & $52(5.7)$ & $22(9.5)$ & 4.408 & 0.036 \\
Nonfatal MI(\%) & $12(1.3)$ & $9(3.9)$ & 6.774 & 0.009 \\
Revascularization(\%) & $37(4.1)$ & $12(5.2)$ & 0.571 & 0.450 \\
Heart failure(\%) & $14(1.5)$ & $4(1.7)$ & - & 0.771 \\
All-cause mortality(\%) & $50(5.5)$ & $27(11.7)$ & 11.231 & 0.001 \\
\hline
\end{tabular}

Nonfatal MI: nonfatal myocardial infarction

Table 3. Logistic regression analysis of the effect of CKD on clinical outcome events (before and after adjusted)

\begin{tabular}{lcccccccc}
\hline & \multicolumn{3}{c}{ Unadjusted adjusted } & \multicolumn{3}{c}{ adjusted } \\
& OR & \multicolumn{2}{c}{$95 \%$ CI } & $p$ & OR & $95 \%$ CI & $p$ \\
\hline Stroke(\%) & 1.737 & 1.032 & 2.924 & 0.038 & 1.694 & 0.967 & 2.966 & 0.065 \\
Nonfatal MI(\%) & 3.034 & 1.263 & 7.290 & 0.013 & 2.675 & 1.023 & 6.995 & 0.045 \\
Revascularization(\%) & 1.293 & 0.663 & 2.521 & 0.451 & 1.664 & 0.808 & 3.427 & 0.167 \\
Heart failure(\%) & 1.128 & 0.368 & 3.459 & 0.833 & 1.399 & 0.418 & 4.682 & 0.586 \\
All- cause mortality(\%) & 2.276 & 1.391 & 3.725 & 0.001 & 1.833 & 1.079 & 3.114 & 0.025 \\
\hline
\end{tabular}

Nonfatal MI: nonfatal myocardial infarction 